\title{
Integration approach of the Couette inverse problem of powder type self-compacting concrete in a wide-gap concentric cylinder rheometer
}

\author{
G. Heirman ${ }^{\mathrm{a}, *}$, L. Vandewalle ${ }^{\mathrm{a}}$, D. Van Gemert ${ }^{\mathrm{a}}$, Ó. Wallevik ${ }^{\mathrm{b}}$ \\ ${ }^{a}$ Reyntjens Laboratory, Department of Civil Engineering, K.U. Leuven, Belgium \\ ${ }^{\mathrm{b}}$ Rheocenter, Icelandic Building Research Institute (IBRI), Iceland \\ Received 6 April 2007; received in revised form 11 September 2007; accepted 6 October 2007
}

\begin{abstract}
For powder type self-compacting concrete (SCC) mixes, commonly used in Belgium, a shear thickening (Herschel-Bulkley) flow behaviour of the fresh mixes is quite often observed.

A longstanding problem in rheometry is the so-called "Couette inverse problem", where one tries to derive the flow curve $\tau(\dot{\gamma})$ from the torque measurements $T(N)$ in a (wide-gap) concentric cylinder (Couette) rheometer, with $T$ the torque registered at the inner, stationary cylinder and $N$ the rotational velocity of the outer, rotating, cylinder.

In this paper, the Couette inverse problem is approached by means of the integration method in order to convert $T(N)$ into $\tau(\dot{\gamma})$ for a wide-gap $\left(R_{\mathrm{o}} / R_{\mathrm{i}}=1.45\right)$ concentric cylinder rheometer. The approach consists in the decoupling of the flow resistance and the power-law flow behaviour after exceeding the flow resistance. The integration approach is validated by experimental verification with different powder type SCC mixtures. By means of illustration, the results of one limestone powder type SCC mixture with different superplasticizer contents are shown in this paper. (C) 2007 Elsevier B.V. All rights reserved.
\end{abstract}

Keywords: Self-compacting concrete; Couette inverse problem; Integration approach; Shear thickening; Herschel-Bulkley

\section{Introduction to self-compacting concrete (SCC)}

Placement of "traditionally vibrated" concrete (TC) requires compaction by vibration in its forms. However, durability problems related to bad compaction quality led to the introduction of self-compacting concrete in Japan at the beginning of the 1980's [1]. SCC is able to flow and consolidate under its own weight, completely filling the formwork even in the presence of dense reinforcement, whilst maintaining homogeneity and without the need for any additional compaction effort [2]. In order to comply with this definition, fresh SCC mixes must possess following key properties:

\footnotetext{
* Corresponding author at: Kasteelpark Arenberg 40 b2448, 3001 Heverlee, Belgium. Fax: +32 16321976 .

E-mail address: Gert.Heirman@bwk.kuleuven.be (G. Heirman).
}

1. Filling ability: The ease of flow of fresh concrete when unconfined by formwork and/or reinforcement.

2. Passing ability: The ability of fresh concrete to flow through tight openings such as spaces between steel reinforcing bars without segregation or blocking.

3. Resistance to segregation: The ability of concrete to remain homogeneous in composition while in its fresh state.

The first two properties, i.e. a sufficiently high flowability, are achieved by using a high range water reducing (HRWR) admixture, mostly based on (modified) polyacrylates (PA) or polycarboxylate ethers (PCE), also called a "3rd generation" superplasticizer. In order to achieve a sufficiently high resistance to segregation, the viscosity of the mixture must be increased compared to TC. This can be done in three ways, mostly depending on readily available materials: (1) using a higher powder content: "powder type SCC", (2) using a viscosity modifying agent (VMA): "VMA type SCC", or (3) using both a higher powder content and VMA: "Combination type SCC". Besides 


\begin{tabular}{|c|c|}
\hline \multicolumn{2}{|c|}{ Nomenclature } \\
\hline B & Bingham \\
\hline $\mathrm{C} / \mathrm{P}$ & cement/powder ratio by mass \\
\hline$D_{\max }$ & maximum aggregate size $(\mathrm{mm})$ \\
\hline$G^{*}$ & (complex) shear modulus $(\mathrm{Pa})$ \\
\hline$G_{\mathrm{B}}$ & flow resistance of B fluids ( $\mathrm{N} \mathrm{m}$ ) \\
\hline$G_{\mathrm{HB}}$ & flow resistance of HB fluids $(\mathrm{N} \mathrm{m})$ \\
\hline$\Delta G_{\mathrm{HB}}$ & $\begin{array}{l}\text { 95\% confidence interval half-width for } G_{\mathrm{HB}} \\
(\mathrm{N} \mathrm{m})\end{array}$ \\
\hline$H_{\mathrm{B}}$ & flow resistance of B fluids $(\mathrm{N} \mathrm{m})$ \\
\hline$H_{\mathrm{HB}}$ & flow resistance of $\mathrm{HB}$ fluids $(\mathrm{Nm})$ \\
\hline$\Delta H_{\mathrm{HB}}$ & $\begin{array}{l}95 \% \text { confidence interval half-width for } H_{\mathrm{HB}} \\
(\mathrm{N} \mathrm{m})\end{array}$ \\
\hline HB & Herschel-Bulkley \\
\hline$h$ & height of the inner cylinder (m) \\
\hline$J$ & flow index factor of HB fluids $(-)$ \\
\hline$\Delta J$ & $95 \%$ confidence interval half-width for $J(-)$ \\
\hline$K$ & consistency coefficient $\left(\mathrm{Pa} \mathrm{s}^{n}\right)$ \\
\hline$\Delta K$ & $95 \%$ confidence interval half-width for $K\left(\mathrm{~Pa} \mathrm{~s}^{n}\right)$ \\
\hline$n$ & flow index $(-)$ \\
\hline$\Delta n$ & $95 \%$ confidence interval half-width for $n(-)$ \\
\hline$N$ & angular velocity of the outer cylinder (rps) \\
\hline$N_{\mathrm{p}}$ & $\begin{array}{l}\text { angular velocity beneath which a plug is formed } \\
\text { in the test material (rps) }\end{array}$ \\
\hline Od & Oldroyd number $(-)$ \\
\hline PCE & polycarboxylate ether (superplasticizer) \\
\hline$r$ & radial cylindrical coordinate $(\mathrm{m})$ \\
\hline$R_{\mathrm{i}}$ & external radius of the inner cylinder $(=0.100 \mathrm{~m})$ \\
\hline$R_{\mathrm{O}}$ & internal radius of the outer cylinder $(=0.145 \mathrm{~m})$ \\
\hline$R_{\mathrm{p}}$ & $\begin{array}{l}\text { plug radius, indicating the boundary between vis- } \\
\text { coplastic and solid state of the test material when } \\
\text { a plug is formed }(\mathrm{m})\end{array}$ \\
\hline SF & slump-flow $(\mathrm{mm})$ \\
\hline SP & superplasticizer \\
\hline$T$ & torque measured on inner cylinder $(\mathrm{N} \mathrm{m})$ \\
\hline TW & time after water addition $(\min )$ \\
\hline$U$ & velocity of suspended particle $(\mathrm{m} / \mathrm{s})$ \\
\hline$v_{\theta}$ & tangential velocity $(\mathrm{m} / \mathrm{s})$ \\
\hline$V_{\mathrm{m}}$ & matrix volume $(1)$ \\
\hline$V_{\mathrm{P}}$ & volume of suspended particles ( $>2 \mathrm{~mm})(1)$ \\
\hline $\mathrm{W} / \mathrm{C}$ & water/cement ratio by mass \\
\hline $\mathrm{W} / \mathrm{P}$ & water/powder ratio by mass \\
\hline$z$ & height cylindrical coordinate $(\mathrm{m})$ \\
\hline \multicolumn{2}{|c|}{ Greek letters } \\
\hline$\Phi$ & $\begin{array}{l}\text { initial particle volume fraction }(-) \\
{\left[=V_{\mathrm{P}} /\left(V_{\mathrm{P}}+V_{\mathrm{m}}\right)\right]}\end{array}$ \\
\hline$\dot{\gamma}$ & shear rate $\left(\mathrm{s}^{-1}\right)$ \\
\hline$\mu$ & plastic viscosity of B fluids (Pa s) \\
\hline$\theta$ & angular cylindrical coordinate (rad) \\
\hline
\end{tabular}

\begin{tabular}{|ll|}
\hline & \\
$\tau_{0, \mathrm{~B}}$ & yield stress of B fluids $(\mathrm{Pa})$ \\
$\tau_{0, \mathrm{HB}}$ & yield stress of HB fluids $(\mathrm{Pa})$ \\
$\Delta \tau_{0, \mathrm{HB}}$ & $95 \%$ confidence interval half-width for $\tau_{0, \mathrm{HB}}(\mathrm{Pa})$ \\
$\omega$ & angular velocity $(\mathrm{rad} / \mathrm{s})\left[=v_{\theta} / r\right]$ \\
$\Omega_{\mathrm{O}}$ & angular velocity of the outer cylinder $(\mathrm{rad} / \mathrm{s})$ \\
& {$[=2 \pi N]$} \\
& \\
$\hat{F}_{\text {unction }}$ & \\
& LerchPhi function \\
Others & \\
$\mathbb{N}$ & set of natural numbers \\
$\infty$ & infinity \\
\end{tabular}

cement, the European "powder type SCC" (having a total powder content of about $550-650 \mathrm{~kg} / \mathrm{m}^{3}$ ) makes mostly use of mineral additions like limestone powder, quartzite powder, fly ash or silica fume in order to reduce heat generation during cement hydration. The (North American) "VMA type SCC" makes use of a VMA, mostly based on polyethylene-glycol or biopolymers (like welan gum) and has a total powder content of about $350-450 \mathrm{~kg} / \mathrm{m}^{3}$. The "combination type SCC" makes use of both a VMA and a total powder content of about 450 $550 \mathrm{~kg} / \mathrm{m}^{3}$.

The SCC mixture used in this paper is based on the "powder type SCC" philosophy, using in Belgium readily available materials.

The flow behaviour of fresh SCC is mostly characterized by empirical test methods like slump-flow, V-funnel, L-box, sieve stability, etc. [2,3]. Because test equipments are inexpensive and tests are carried out easily, they are suitable for on site use. However, these tests have one major disadvantage: they provide no fundamental description of flow behaviour. For the knowledge of the flow parameters, rheological measurements from a materials science approach by means of a rheometer, considering the concrete as a fluid (see below), are necessary. A detailed comparison between empirical and rheological data is beyond the scope of this paper.

\section{Rheology and concrete technology}

\subsection{Rheological models in concrete technology}

On beforehand, it should be stated that fresh concrete is treated here as a fluid and, as a consequence, fluid rheology methods are used to describe the concrete flow. In this approach, only the motion of a "large" number of solid particles is taken into consideration, without going into the detailed motion of every single one of them. In order to properly treat fresh concrete as a fluid, a certain degree of flow (i.e. a slump of at least $100 \mathrm{~mm}$, according to EN 12350-2:1999) must be achieved while the concrete stays homogeneous (i.e. no segregation may occur) [4]. 
The rheology of fresh concrete, conceived as a viscoplastic fluid, can usually be described by the Bingham model, at least as a first approximation. In this model, the shear stress $\tau$ is assumed to be linearly proportional to the shear rate $\dot{\gamma}$, after exceeding a certain Bingham yield stress $\tau_{0, \mathrm{~B}}$ :

$\tau= \begin{cases}G^{*} \gamma(\text { or } \dot{\gamma}=0) & \tau<\tau_{0, \mathrm{~B}} \\ \tau_{0, \mathrm{~B}}+\mu \dot{\gamma} & \tau \geq \tau_{0, \mathrm{~B}}\end{cases}$

Below the Bingham yield stress, the fluid behaves Hookean, while it behaves Newtonian above. $G^{*}$ and $\mu$ are called the (complex) shear modulus (Pa) and the plastic viscosity (Pas), respectively. It should be noted that the Bingham yield stress is not necessarily a "true" yield stress [5-7]. The concept of the Bingham (and Herschel-Bulkley, see below) yield stress, however, can be considered as a useful engineering tool in concrete science: it can be seen as the point at which solid-like behaviour is first seen when decreasing the applied stress.

For a considerable number of powder type SCC mixtures, commonly used in Belgium, the Bingham model results in negative values for the Bingham yield stress, which of course has no physical meaning. The effect is more pronounced when higher superplasticizer contents are added to the SCC mix, resulting in a lower yield stress $[8,9]$.

In order to avoid the appearance of a negative yield stress, a shear thickening model must be used to describe this material's flow behaviour. The Herschel-Bulkley approach is mostly used to approximate the shear thickening flow behaviour of concrete [8-12]. In the last model, the relation $\tau(\dot{\gamma})$, after exceeding the Herschel-Bulkley yield stress $\tau_{0, \mathrm{HB}}$, is described by a power-law function:

$\tau= \begin{cases}G^{*} \gamma(\text { or } \dot{\gamma}=0) & \tau<\tau_{0, \mathrm{HB}} \\ \tau_{0, \mathrm{HB}}+K \dot{\gamma}^{n} & \tau \geq \tau_{0, \mathrm{HB}}\end{cases}$

Below the Herschel-Bulkley yield stress, the fluid behaves Hookean, while it is found that the fluid (i.e. the self-compacting concrete mix) behaves dilatant (or shear thickening: $n>1$ ) above the yield value [8]. $K$ is called the "consistency coefficient" $\left(\mathrm{Pa} \mathrm{s}^{n}\right)$, where $n$ denotes the "flow index" $(-)$.

According to Cyr et al. [12], the shear thickening behaviour of cement pastes could be linked (besides to the high polydispersity of the particles [13]) to the presence of a superplasticizer. Two possible explanations are given: (1) increasing the shear rate enhances the disorder, not only between the cement particles, but also within the polymeric chains of the superplasticizer, and thus increases the viscosity (see Hoffman [14-16] for more information on the order-disorder theory); (2) increasing the shear rate can locally tear off part of the absorbed polymer, resulting in (a) an increased viscosity of the free water, and so possibly making it shear thickening (according to Hoffman's theory), and in (b) a higher probability of occurrence of interparticular bonds and flocs (which results in an increased viscosity, according to Bossis and Brady's clustering theory [17-19]).

Mineral additions as replacements of different amounts of cement modify the intensity of shear thickening (indi-

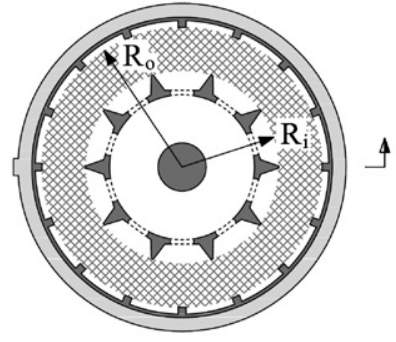

(a) Horizontal section

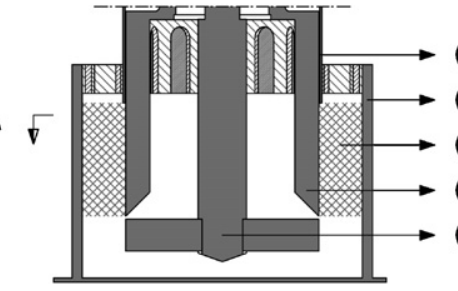

Fig. 1. Schematic cross-section of the ConTec Visco5: (1) top ring; (2) outer cylinder, internal radius $R_{0}=145 \mathrm{~mm}$; (3) sheared test material taken into consideration; (4) inner cylinder - free upper unit, external radius $R_{\mathrm{i}}=100 \mathrm{~mm}$; (5) inner cylinder - fixed bottom unit.

cated by the flow index $n$ ), depending on their nature: the phenomenon can be increased, unaltered or decreased compared to plain cement pastes [12]. Assuming an unaltered intensity for quartz powder "Silfill 3600 M" from Sibelco, Belgium (as found in [12]), the use of limestone powder ("Calcitec 2001 S" from Carmeuse, Belgium, and "Betocarb P2 Mq" from Omya Industries Inc., France) as mineral addition for powder type SCC results in a (slightly) amplified intensity, while fly ash (from Electrabel power station "Langerlo", Belgium) seems to reduce the shear thickening intensity [8].

\subsection{The Couette concentric cylinder rheometer in concrete technology}

The "ConTec Visco5" used for the experiments in this paper can be considered as an example of a Couette concentric cylinder rheometer (Figs. 1 and 2). It is one of the most recent updates of the BML viscometer [20], well designed for testing both (self-compacting) concrete and mortar (maximum aggregate size $D_{\max } \leq 22 \mathrm{~mm}$ ). Most important improvements of the

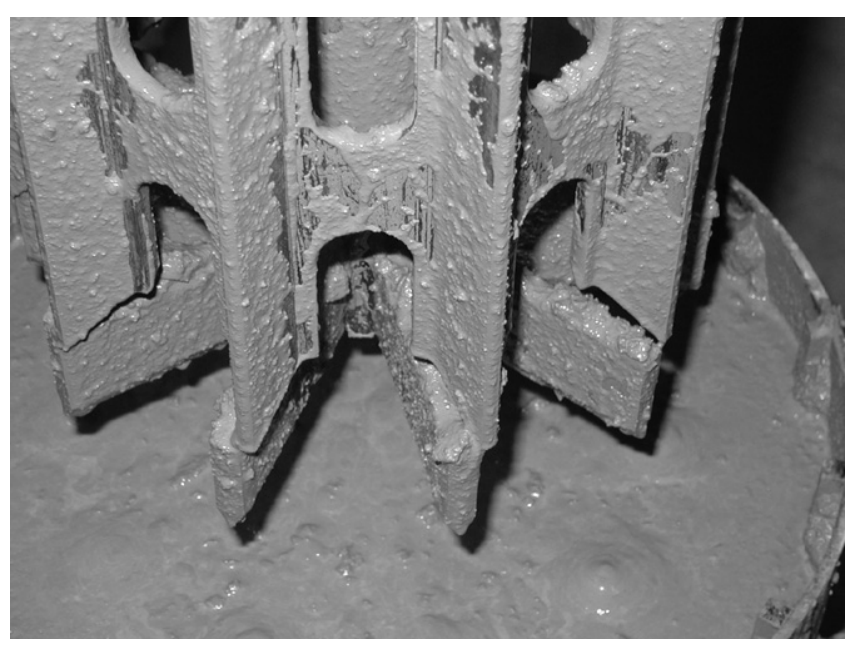

Fig. 2. Detailed view of inner cylinder upper and bottom unit of the ConTec Visco5 (here: immediately after testing a SCC mixture). 
ConTec Visco5 are: increased torque signal sensitivity, reduction of signal noise during torque logging, increased rotation speed span and increased criteria for accuracy of the centre axes of the inner and outer cylinder, together with some minor modifications on the inner cylinder. The outer cylinder rotates at angular velocity $\Omega_{\mathrm{o}}=2 \pi N(\mathrm{rad} / \mathrm{s})$, while the inner cylinder is stationary and registers the applied torque $T(\mathrm{Nm})$ from the test material, i.e. in our case the self-compacting concrete.

The measuring system consists of an outer cylinder $\left(R_{\mathrm{O}}=145 \mathrm{~mm}\right)$, an inner cylinder unit (standard unit C-200: $R_{\mathrm{i}}=100 \mathrm{~mm}$ ) and a top ring. To avoid slippage between the cylinders and the test material, both the inner and outer cylinders are fitted with protruding vanes (Fig. 1a) [21-23]. With this, the dimensions $R_{\mathrm{i}}$ and $R_{\mathrm{O}}$ are relative to the extremities of the vanes (Fig. 1a). For a viscoplastic material, it is assumed that the material is held in the space between the vane blades so that it behaves like a rigid cylinder. Experimental observation supports this assumption and is in accordance with the experiences found in literature [23-26]. Any attempt to investigate the flow between the vane blades is not done in this work.

The outer cylinder is well mounted on a rotating disk. The inner cylinder is lowered into the outer cylinder by a screw-jack system. The top ring can be fitted over the inner cylinder, in order to insure a constant height of the sheared test material. The inner cylinder is constructed as a two component unit (Figs. 1b and 2): a bottom unit, which is fixed at the mounting point of the inner cylinder, and an upper unit, which is free to rotate against a load cell, registering the applied torque $T$ from the rheological continuum. The arrangement of the two component inner cylinder will virtually eliminate the effect of $3 \mathrm{D}$ shearing at the bottom of the inner cylinder and therefore requires no special correction regarding possible bottom effects [27].

\section{Shear rate and shear stress in a wide-gap concentric cylinder rheometer}

The shear rate in a rotational flow is defined as:

$\dot{\gamma}=r \frac{\partial \omega(r)}{\partial r}$

with $r$ the radial cylindrical coordinate $(m)$ and $\omega(r)$ the angular velocity at radius $r(\mathrm{rad} / \mathrm{s})$. A detailed derivation of this equation can be found elsewhere [27,28]. In short, the following assumptions are made concerning the velocity profile in order to obtain Eq. (3):

- The flow between the two concentric cylinders is stable (i.e. laminar, without secondary flows) and symmetrical in $z$-direction.

- The velocity profile is height independent because bottom and top effects are eliminated by geometrical means (fixed bottom unit of inner cylinder and top ring).

- The flow is purely circular with angle independence, due to the circular geometry of the Couette rheometer.
- The flow is steady state, i.e. time independent for the given measurement, because each measurement is applied in equilibrium conditions (equilibrium torque at each rotational velocity).

The shear stress applied from the test material on the inner cylinder can be determined from the decomposition of the conservation of momentum in $\theta$-direction and with the help of Cauchy's stress principle, expressing equivalence between applied torque and shear stresses on the shell $[27,28]$ :

$\tau(r)=\frac{T}{2 \pi r^{2} h}$

with $T$ the measured torque $(\mathrm{Nm})$ and $h$ the height of the considered cylindrical shell $(\mathrm{m})$. The latter equation shows that the shear stress of a fluid in a concentric rheometer depends only on the rheometer geometry and not on the nature of the fluid (at steady state).

The derivation of the flow curve $\tau(\dot{\gamma})$ from the torque measurements $T(N)$ is called the "Couette inverse problem". In order to solve the Couette inverse problem, three methods can be used: (a) the integration method: the type of constitutive equation is specified in advance and integrated to obtain the relation $T(N)$, which is fitted to the experimental data, (b) the Tikhonov regularization method, proposed by Yeow et al. [29] and (c) the wavelet-vaguelette decomposition method, proposed by Ancey [30].

The analytical solution of the Couette inverse problem is up to now reserved for special fluid rheological behaviour, i.e. when using a narrow-gap rheometer geometry. For wide-gap concentric cylinder rheometry, as it is the case for (self-compacting) concrete, a straight analytical solution is not available up till now [31,32].

\section{Integration approach of the Couette inverse problem for a Bingham fluid: the "Reiner-Riwlin" equation}

Eq. (1), for $\tau \geq \tau_{0, \mathrm{~B}}$, can be rewritten, using Eqs. (3) and (4), as:

$\frac{T}{2 \pi r^{2} h}=\tau_{0, \mathrm{~B}}+\mu r \frac{\partial \omega(r)}{\partial r}$

Assuming "no slip" boundary conditions, i.e. $r=R_{\mathrm{i}} \Rightarrow \omega \equiv 0 \wedge r=R_{\mathrm{O}} \Rightarrow \omega \equiv \Omega_{\mathrm{o}}$, the above equation can be integrated as follows:

$\int_{R_{\mathrm{i}}}^{R_{\mathrm{O}}}\left(\frac{T}{2 \pi r^{3} h}-\frac{\tau_{0, \mathrm{~B}}}{r}\right) \mathrm{d} r=\mu \int_{0}^{\Omega_{\mathrm{o}}} \mathrm{d} \omega(r)$ 
The solution of this integral (see Appendix A) results in the well-known "Reiner-Riwlin" equation:

$$
T=\frac{4 \pi h \tau_{0, \mathrm{~B}}}{\left(\frac{1}{R_{\mathrm{i}}^{2}}-\frac{1}{R_{\mathrm{o}}^{2}}\right)} \ln \left(\frac{R_{\mathrm{o}}}{R_{\mathrm{i}}}\right)+\frac{\mu 8 \pi^{2} h}{\left(\frac{1}{R_{\mathrm{i}}^{2}}-\frac{1}{R_{\mathrm{o}}^{2}}\right)} N \equiv G_{\mathrm{B}}+H_{\mathrm{B}} N
$$

where $G_{\mathrm{B}}$ and $H_{\mathrm{B}}$ are, respectively, the flow resistance and the viscosity factor for a Bingham fluid. So:

$\tau_{0, \mathrm{~B}}=\frac{G_{\mathrm{B}}}{4 \pi h}\left(\frac{1}{R_{\mathrm{i}}^{2}}-\frac{1}{R_{\mathrm{o}}^{2}}\right) \frac{1}{\ln \left(R_{\mathrm{o}} / R_{\mathrm{i}}\right)}$

and

$\mu=\frac{H_{\mathrm{B}}}{8 \pi^{2} h}\left(\frac{1}{R_{\mathrm{i}}^{2}}-\frac{1}{R_{\mathrm{o}}^{2}}\right)$

Eq. (7) is the solution of the inverse (wide-gap) Couette problem for a Bingham fluid, since the following steps can be made in order to derive the flow curve $\tau(\dot{\gamma})$ from the torque measurements $T(N)$ :

- Determine $G_{\mathrm{B}}$ and $H_{\mathrm{B}}$ by a linear least square curve fitting of the experimental data $T(N)$ into $T=G_{\mathrm{B}}+H_{\mathrm{B}} N$ (keeping in mind that a steady state flow and no appearance of slip nor plug are required).

- Determine $\tau_{0, \mathrm{~B}}$ and $\mu$, according to Eqs. (8) and (9), respectively, and fill in their values into Eq. (1) (for $\tau \geq \tau_{0, \mathrm{~B}}$ ).

When changing the "outer" boundary condition into $r^{\prime}=r \Rightarrow \omega \equiv v_{\theta}(r) / r$, Eq. (6) can be solved for the velocity profile in the gap of the Couette rheometer:

$v_{\theta}(r)=\frac{T r}{4 \pi h \mu}\left(\frac{1}{R_{\mathrm{i}}^{2}}-\frac{1}{r^{2}}\right)-\frac{\tau_{0, \mathrm{~B}} r}{\mu} \ln \left(\frac{r}{R_{\mathrm{i}}}\right)$

Using Eq. (3), the corresponding shear rate can be calculated as:

$\dot{\gamma}(r)=\frac{T}{4 \pi r^{2} h \mu}-\frac{\tau_{0, \mathrm{~B}}}{\mu}$

It may be clear from the latter two equations that, contrary to the shear stress (Eq. (4)), the velocity profile and shear rate depend on both the rheometer geometry and the nature of the fluid (at steady state).

It should be noted that the boundary conditions, as mentioned in Eq. (6), are only valid when no plug is formed during the rheological measurements. With a plug, a solid state arises $(\dot{\gamma} \equiv$ $0)$. The material in the solid state rotates inside the rheometer as a rigid body $\left(v_{\theta}(r)=r \Omega_{\mathrm{o}}\right)$. When applying a stepwise decreasing rotational speed sequence (see Section 6.3), the condition $\dot{\gamma}=$ 0 , and so plug, will begin at the outer cylinder and propagate towards the inner cylinder as the angular velocity $\Omega_{0}(=2 \pi N)$ is further decreased [27]. The location of the boundary between the viscoplastic and the solid state is defined by the plug radius
$R_{\mathrm{p}}$, which can be calculated as:

$R_{\mathrm{p}}=\sqrt{\frac{T}{2 \pi h \tau_{0, \mathrm{~B}}}}$

For $R_{\mathrm{p}}<R_{0}$, i.e. when a plug is present, the outer boundary condition of Eq. (6) must be replaced by " $r=R_{\mathrm{p}} \Rightarrow \omega \equiv \Omega_{\mathrm{o}}$ ", although this latter condition may be incorrect due to possible slippage in the transition zone from viscoplastic to solid state. When slippage occurs, the rheometer measures a smaller torque than expected.

\section{Integration approach of the Couette inverse problem for a Herschel-Bulkley fluid}

\subsection{Integration approach of the Couette inverse problem for $T=G_{H B}+H_{H B} N^{J}$}

Since Eqs. (3) and (4) are not depending on any rheological model, they can also be used in the case of a Herschel-Bulkley fluid. So Eq. (2), for $\tau \geq \tau_{0, \mathrm{HB}}$, can be rewritten as:

$\frac{T}{2 \pi r^{2} h}=\tau_{0, \mathrm{HB}}+K\left(r \frac{\partial \omega(r)}{\partial r}\right)^{n}$

The same "no slip" boundary conditions as mentioned for the Bingham fluid can be applied, resulting in the following integration of Eq. (13):

$\int_{R_{\mathrm{i}}}^{R_{\mathrm{o}}}\left(\left(\frac{T}{2 \pi r^{2} h K}-\frac{\tau_{0, \mathrm{HB}}}{K}\right)^{1 / N} \frac{1}{r}\right) \mathrm{d} r=\int_{0}^{\Omega_{0}} \mathrm{~d} \omega(r)$

Unfortunately, the solution of this integral (see Appendix B) contains the LerchPhi function $\hat{\phi}(x, 1, a)$ which is a singlevalued, continuous but non-polynomial function on the $x$-plane cut along the interval $x \in]-\infty, 1[$ (for fixed $a,-a \notin \mathbb{N}$ ) [33]:

$$
\begin{aligned}
& {\left[\begin{array}{l}
\left(\frac{T}{2 \pi R_{\mathrm{i}}^{2} h K}-\frac{\tau_{0, \mathrm{HB}}}{K}\right)^{1 / n}\left[n-\hat{\phi}\left(1-\frac{T}{2 \pi R_{\mathrm{i}}^{2} h \tau_{0, \mathrm{HB}}}, 1, \frac{1}{n}\right)\right]- \\
\left(\frac{T}{2 \pi R_{\mathrm{o}}^{2} h K}-\frac{\tau_{0, \mathrm{HB}}}{K}\right)^{1 / n}\left[n-\hat{\phi}\left(1-\frac{T}{2 \pi R_{\mathrm{o}}^{2} h \tau_{0, \mathrm{HB}}}, 1, \frac{1}{n}\right)\right]
\end{array}\right]} \\
& =4 \pi N
\end{aligned}
$$

Similar to the Bingham fluid, the latter equation should preferably be transformed into the general form:

$T=G_{\mathrm{HB}}+H_{\mathrm{HB}} N^{J}$

with $G_{\mathrm{HB}}$ the flow resistance, $H_{\mathrm{HB}}$ the viscosity factor and $J$ the flow index factor for a Herschel-Bulkley fluid.

For $n=1$, the applicability of Eq. (15) is established by Heirman et al. [28], resulting in the same "Reiner-Riwlin" equation as found above (Eq. (7)). However, for $n \neq 1$, no analytical transformation into the general form can be found, due to the non-polynomial character of the LerchPhi function.

Note that, similar to a Bingham fluid and applying the same outer boundary condition $r^{\prime}=r \Rightarrow \omega \equiv v_{\theta}(r) / r$, the velocity dis- 
tribution across the gap is given by:

$v_{\theta}(r)=\frac{r}{2}\left[\begin{array}{l}\left(\frac{T}{2 \pi R_{\mathrm{i}}^{2} h K}-\frac{\tau_{0, \mathrm{HB}}}{K}\right)^{1 / n}\left[n-\hat{\phi}\left(1-\frac{T}{2 \pi R_{\mathrm{i}}^{2} h \tau_{0, \mathrm{HB}}}, 1, \frac{1}{n}\right)\right]- \\ \left(\frac{T}{2 \pi r^{2} h K}-\frac{\tau_{0, \mathrm{HB}}}{K}\right)^{1 / n}\left[n-\hat{\phi}\left(1-\frac{T}{2 \pi r^{2} h \tau_{0, \mathrm{HB}}}, 1, \frac{1}{n}\right)\right]\end{array}\right]$

It should be noted that also for a Herschel-Bulkley fluid, steady state flow and no appearance of slip or plug are required in order to fulfil the boundary conditions as given in Eq. (14). In case of plug, the same outer boundary condition correction as seen for a Bingham fluid (Section 4) can be made (see also Section 6.3).

Experimental results, including the $95 \%$ confidence intervals for the model parameters $\left(G_{\mathrm{HB}}, H_{\mathrm{HB}}\right.$ and $\left.J\right)$, the predicted model $T(N)$ and the unknown torque $T$, for different powder type SCC mixtures can be found in [8]. Besides, experiments revealed that the integration approach of the Couette inverse problem and the superposition of a flow resistance with a power-law flow behaviour are "commutative": a reliable approach of the Couette inverse problem of the additive function $T=G_{\mathrm{HB}}+H_{\mathrm{HB}} N^{J}$ can be found as the sum of the solutions of the Couette inverse problem of both subfunctions $T^{\prime}=G_{\mathrm{HB}}$ (the flow resistance) and $T^{\prime \prime}=H_{\mathrm{HB}} N^{J}$ (the power-law, Ostwald-de Waele, flow behaviour after exceeding the flow resistance).

The experiments proved that the "decoupling of terms" could be extended from the linear to the nonlinear case for (see [8] for all experimental results): $R_{\mathrm{O}} / R_{\mathrm{i}}=1.45 ; 1<J<1.81$ and $1.9 \cdot 10^{-4}<O d<33.4$. Od is the Oldroyd number, expressing the ratio of the plastic, Herschel-Bulkley yield, stress to viscous stresses, given by:

$O d=\frac{\tau_{0, \mathrm{HB}}}{K(U / d)^{n}}$

with $d$ the suspended particle diameter (m) and $U$ its (tangential) velocity $(\mathrm{m} / \mathrm{s})$.

The maximum diameter is given by the maximum aggregate size $D_{\max }$ (= $14 \mathrm{~mm}$ for all SCC mixtures under consideration). The minimum diameter is arbitrary set at $2 \mathrm{~mm}$. It should be noted that, dealing with fresh concrete, the distinction between matrix and suspended particles is in fact a matter of choice, in contrast to the more traditional suspensions of spheres submerged in a Newtonian liquid. For concrete, the matrix is defined here to be the $0-2 \mathrm{~mm}$ mortar inside it, in accordance with Wallevik [27] and Barnes et al. [34].

In Sections 5.2 and 5.3, the integration approach for the Couette inverse problem of each subfunction will be given. In Section 5.4 , the integration approach for obtaining the flow curve $\tau(\dot{\gamma})$ of a Herschel-Bulkley fluid is presented.

\subsection{Integration approach of the Couette inverse problem for the flow resistance $T^{\prime}=G_{H B}$}

For $T^{\prime}=G_{\mathrm{HB}}$, experiments proved that the same conversion equation as found for the flow resistance of a Bingham fluid (Eq. (8)) can be used. Besides, it was found before that the shear stress of a fluid depends only on the rheometer geometry and not on the nature of the fluid:

$\tau_{0, \mathrm{HB}}=\frac{G_{\mathrm{HB}}}{4 \pi h}\left(\frac{1}{R_{\mathrm{i}}^{2}}-\frac{1}{R_{\mathrm{o}}^{2}}\right) \frac{1}{\ln \left(R_{\mathrm{o}} / R_{\mathrm{i}}\right)}$

\subsection{Integration approach of the Couette inverse problem} for $T^{\prime \prime}=H_{H B} N^{J}$

In the case of no flow resistance $\left(G_{\mathrm{HB}} \equiv 0\right)$, the constitutive equation to be specified in advance simplifies to the (Ostwald-de Waele) power-law model:

$\frac{T^{\prime \prime}}{2 \pi r^{2} h}=K\left(r \frac{\partial \omega(r)}{\partial r}\right)^{n}$

Also here applying the same "no slip" boundary conditions as mentioned for the Bingham fluid, the following integration of Eq. (20) is found:

$\int_{R_{\mathrm{i}}}^{R_{\mathrm{o}}}\left(\left(\frac{T^{\prime \prime}}{2 \pi r^{2} h K}\right)^{1 / n} \frac{1}{r}\right) \mathrm{d} r=\int_{0}^{\Omega_{\mathrm{o}}} \mathrm{d} \omega(r)$

The solution of this integral (see Appendix C) results in:

$T^{\prime \prime}=\frac{2^{2 n+1} \pi^{n+1} h K}{n^{n}\left(1 / R_{\mathrm{i}}^{2 / n}-1 / R_{\mathrm{o}}^{2 / n}\right)^{n}} N^{n} \equiv H_{\mathrm{HB}} N^{J}$

So:

$n=J$

and

$K=\frac{H_{\mathrm{HB}}}{2^{2 n+1} \pi^{n+1} h} n^{n}\left(\frac{1}{R_{\mathrm{i}}^{2 / n}}-\frac{1}{R_{\mathrm{o}}^{2 / n}}\right)^{n}$

5.4. Integration approach of the Couette inverse problem for $T=T^{\prime}+T^{\prime \prime} \equiv G_{H B}+H_{H B} N^{J}$

As stated above, the integration approach for the Couette inverse problem of the additive function $T=G_{\mathrm{HB}}+H_{\mathrm{HB}} N^{J}$ consists of the sum of the solutions of the Couette inverse problem of both $T^{\prime}=G_{\mathrm{HB}}$ and $T^{\prime \prime}=G_{\mathrm{HB}} N^{J}$. In this way, a reliable approach of the ultimate flow curve $\tau(\dot{\gamma})$, after exceeding the Herschel-Bulkley yield stress $\tau_{0, \mathrm{HB}}$, can be derived from the torque measurements $T(N)$ as follows:

- Determine $G_{\mathrm{HB}}, H_{\mathrm{HB}}$ and $J$ by a nonlinear least square curve fitting of the experimental data $T(N)$ into $T=G_{\mathrm{HB}}+H_{\mathrm{HB}} N^{J}$ 
(keeping in mind that a steady state flow and no appearance of slip nor plug are required).

- Determine $\tau_{0, \mathrm{HB}}$, according to Eq. (19).

- Determine $n$ and $K$, according to Eq. (23) and (24), respectively, for $T^{\prime \prime}=T-G_{\mathrm{HB}}=H_{\mathrm{HB}} N^{J}$.

- Fill in the values of $\tau_{0, \mathrm{HB}}, n$ and $K$ into Eq. (2) (for $\tau \geq \tau_{0, \mathrm{HB}}$ ).

Similar to the "Reiner-Riwlin" equation, the analytical solution for a Herschel-Bulkley fluid can also be expressed as:

$$
\begin{aligned}
T= & \frac{4 \pi h \tau_{0, \mathrm{HB}}}{\left(1 / R_{\mathrm{i}}^{2}-1 / R_{\mathrm{o}}^{2}\right)} \ln \left(\frac{R_{\mathrm{o}}}{R_{\mathrm{i}}}\right) \\
& +\frac{2^{2 n+1} \pi^{n+1} h K}{n^{n}\left(1 / R_{\mathrm{i}}^{2 / n}-1 / R_{\mathrm{O}}^{2 / n}\right)^{n}} N^{n} \equiv G_{\mathrm{HB}}+H_{\mathrm{HB}} N^{J}
\end{aligned}
$$

Note that Eq. (25) is valid for both shear thickening (or dilatant, $n>1$ ) and shear thinning (or pseudoplastic, $n<1$ ) fluids.

\section{Experimental results}

\subsection{Materials and mix design}

River aggregates (river gravel 4/14 and river sand 0/5), supplied by Gralex (Belgium), are used because of their most favourable, rounded shape for SCC production. They were oven dried before use. The use of a maximum aggregate size $D_{\max }=14 \mathrm{~mm}$ is in accordance with the required minimum gap size $\left(=3 D_{\max }\right)$ for concrete rheometry, provided by Ferraris et al. [4], to avoid interlocking of the aggregates, which will prevent flow (see also [27] for more information about the barrier restraint of inner and outer cylinder). A HeidelbergCement Group (CBR) portland cement (CEM I 52.5 R HES according to EN 197-1:2000) and a Carmeuse finely ground limestone addition (Calcitec 2001 S) are used as "powder". The temperature of the mixing water, having a four to five times higher specific heat capacity than the other mixing materials, was set at $20^{\circ} \mathrm{C}$. As PCE superplasticizer, SIKA ViscoCrete-20 GOLD CON. $40 \%$ was used for the experiments shown in this paper.

The specific gravities of the aggregates and the powders are determined according to EN 1097-6:2000 and EN 1097-7:1999, respectively. The results are listed in Table 1 . The specific gravity of the superplasticizer is taken from its technical data sheet.

Table 1

Specific gravity (SG) of materials (-) and mix composition of SCC24 $\left(\mathrm{kg} / \mathrm{m}^{3}\right)$

\begin{tabular}{lll}
\hline Constituent material & $\mathrm{SG}(-)$ & Composition $\left(\mathrm{kg} / \mathrm{m}^{3}\right)$ \\
\hline River gravel 4/14 & 2.64 & 698 \\
River sand 0/5 & 2.64 & 853 \\
CEM I 52.5 R HES & 3.13 & 360 \\
Limestone addition 1 & 2.70 & 240 \\
Water & 1.00 & 165 \\
Superplasticizer PCE & 1.08 & $4.5+1.5+1.5$ \\
W/C & & 0.46 \\
C/P & & 0.6 \\
W/P & & 0.28 \\
\hline
\end{tabular}

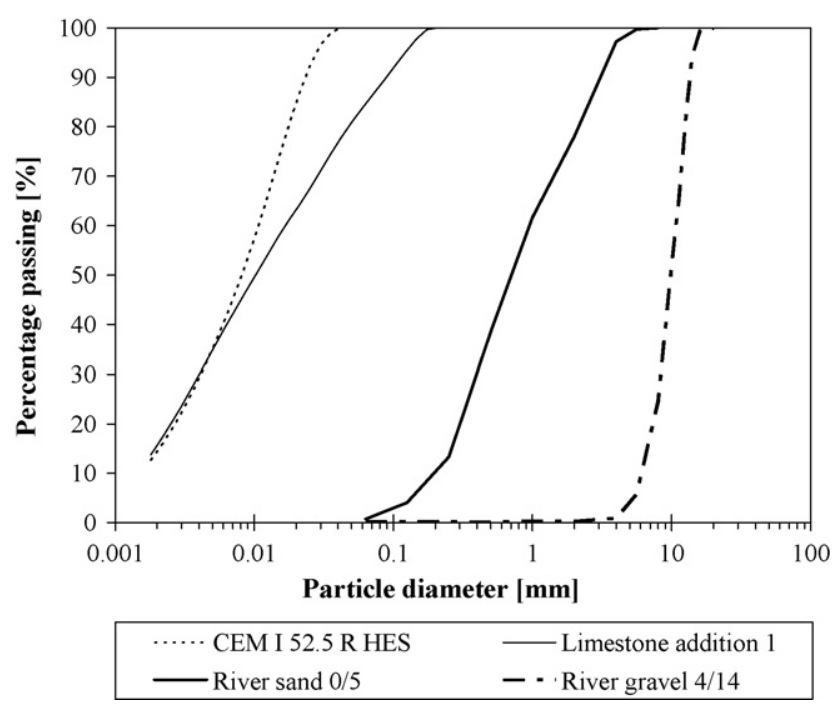

Fig. 3. Grading curves of powders and aggregates for SCC24.

The grading curves of the aggregates are determined according to EN 933-1:1997 and EN 12620:2002, while the grading curves of the powders are determined by means of laser diffraction, using the Sympatec HELOS/QUIXEL apparatus (measuring range 1.8-350 $\mu \mathrm{m}$ ). The results are shown in Fig. 3.

The Blaine fineness, determined according to EN 196-6:1991 and corrected to $20^{\circ} \mathrm{C}$, of both cement and limestone addition are 600 and $338 \mathrm{~m}^{2} / \mathrm{kg}$, respectively.

The mix composition of the SCC mixture under consideration (denoted as SCC24) is given in Table 1. The air content, determined according to EN 12350-7:2000, and the density $\rho$ of the fresh concrete mixture SCC242 are $1.9 \%$ and $2377 \mathrm{~kg} / \mathrm{m}^{3}$, respectively.

\subsection{Mixing procedure}

A 501 batch was mixed in a 50-1 laboratory paddle-pan mixer (EIRICH, type SKG1). First, all the dry material was put in the mixer. Then mixing was started. Water was added during the first $15 \mathrm{~s}$. One minute later, the mixer was paused for $15 \mathrm{~s}$ and the PCE superplasticizer (SP) was added. After SP addition, the mixing continued for $2 \mathrm{~min}$. After mixing, the slump-flow was measured according to the European guidelines for SCC [2, Annex B.1] (using the Abrams cone with internal upper/lower diameter of 100/200 mm and height of $300 \mathrm{~mm}$; base plate of $900 \mathrm{~mm} \times 900 \mathrm{~mm} \times 2 \mathrm{~mm}$, made of stainless steel with smooth and plane surface, deviation of flatness $<3 \mathrm{~mm}$ ) and the rheological properties were determined with the ConTec Visco5 (placed in a climate room at $20 \pm 2{ }^{\circ} \mathrm{C}$ and $54 \pm 5 \%$ R.H.). After testing, the concrete was poured back into the mixer, an extra amount of SP was added and the concrete was mixed for another $2 \mathrm{~min}$. This test sequence is repeated for each further measurement.

For each test under consideration, the amount of SP added (PCE, in $\mathrm{m} \%$, relative to the cement mass), the initial particle volume fraction $(\Phi)$ [34], the time after the addition of water (TW, in $\mathrm{min}$ ) and the slump-flow (SF, in $\mathrm{mm}$; i.e. the average value of the largest diameter of the flow spread of the concrete 
Table 2

PCE-content $(\mathrm{m} \%)$, initial particle volume fraction $\Phi(-)$, time after water addition TW ( $\mathrm{min}$ ) and slump-flow SF (mm) for each rheometry test under consideration

\begin{tabular}{lllcl}
\hline & PCE $(\mathrm{m} \%)$ & $\Phi(-)$ & TW $(\min )$ & SF $(\mathrm{mm})$ \\
\hline SCC242 & 1.25 & 0.473 & 54 & 610 \\
SCC244 & 1.67 & 0.472 & 120 & 690 \\
SCC246 & 2.08 & 0.471 & 182 & 830
\end{tabular}

and the diameter of the spread at right angles to it) are given in Table 2 .

\subsection{Rheological test results and validation of the proposed conversion method}

To obtain the flow curve $T(N)$ with a maximum of steady state data points, the rotational velocity $N$ is decreased in 10 steps $(0.63 \rightarrow 0.06 \mathrm{rps})$ of each $25 \mathrm{~s}$ (100 registration points), after a pre-shearing period of $10 \mathrm{~s}$ at $0.60 \mathrm{rps}$ (in accordance with Wallevik [27], in order to finish thixotropic breakdown, and so to create uniform start conditions for all experiments). For each velocity step, the average of torque $T$ and rotational velocity $N$ measured at the last $15 \mathrm{~s}$ can be seen as one $(T, N)$ data point. If the torque for a certain point did not reach steady state, the point was omitted in further regression analysis. As an illustration, all registered rotational velocity and torque data points for the specific mix SCC242 are shown in Fig. 4 (first point, at $N=0.63 \mathrm{rps}$, omitted in further analysis due to nonsteady state flow).

Curve fitting, according to Eq. (16), of the steady state $(T, N)$ data points is done by means of nonlinear regression analysis, using the stats toolbox of MATLAB ${ }^{\circledR}$ R2006a. For each steady state, the last 60 registration points of both $T$ and $N$ are used for the regression analysis. In the case of Bingham (linear) behaviour, as it is for SCC242 (see below), the flow index factor equals unity: $J \equiv 1$. The results for the model parameters $\left(G_{\mathrm{HB}}\right.$, $H_{\mathrm{HB}}$ and $J$ ) as well as their $95 \%$ confidence interval half-widths are given in Table 3 (only the results for SCC24 are shown here,

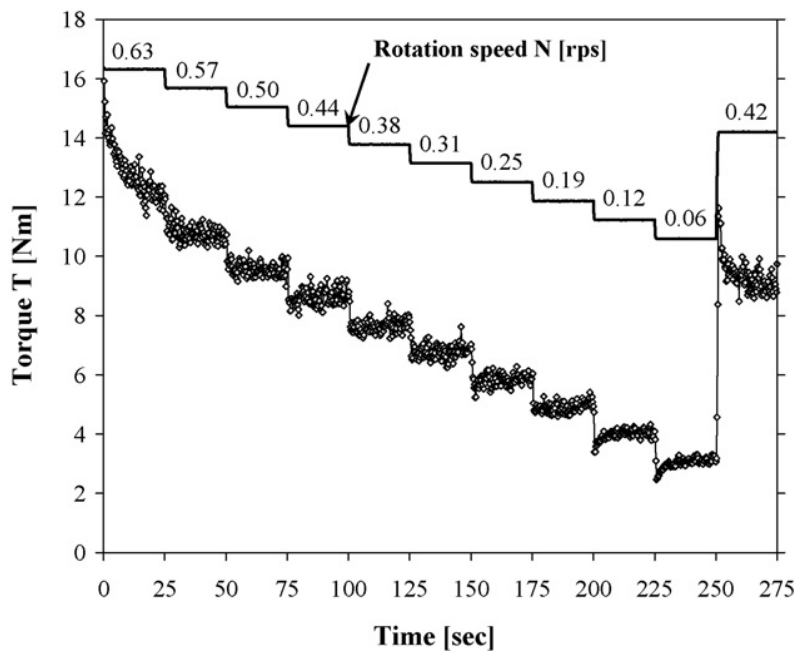

Fig. 4. Registration of rotational velocity and torque data points for SCC242.
Table 3

Results for the $T(N)$ model parameters

\begin{tabular}{lllllll}
\hline & $\begin{array}{l}G_{\mathrm{HB}} \\
(\mathrm{N} \mathrm{m})\end{array}$ & $\begin{array}{l}\Delta G_{\mathrm{HB}} \\
(\mathrm{Nm})\end{array}$ & $\begin{array}{l}H_{\mathrm{HB}} \\
\left(\mathrm{N} \mathrm{m} \mathrm{s}^{J}\right)\end{array}$ & $\begin{array}{l}\Delta H_{\mathrm{HB}} \\
\left(\mathrm{N} \mathrm{m} \mathrm{s}^{J}\right)\end{array}$ & $J(-)$ & $\Delta J(-)$ \\
\hline SCC242 & 2.19 & 0.04 & 14.75 & 0.11 & 1 & 0 \\
SCC244 & 1.07 & 0.06 & 12.38 & 0.14 & 1.30 & 0.03 \\
SCC246 & 0.43 & 0.04 & 10.70 & 0.17 & 1.65 & 0.03 \\
\hline
\end{tabular}

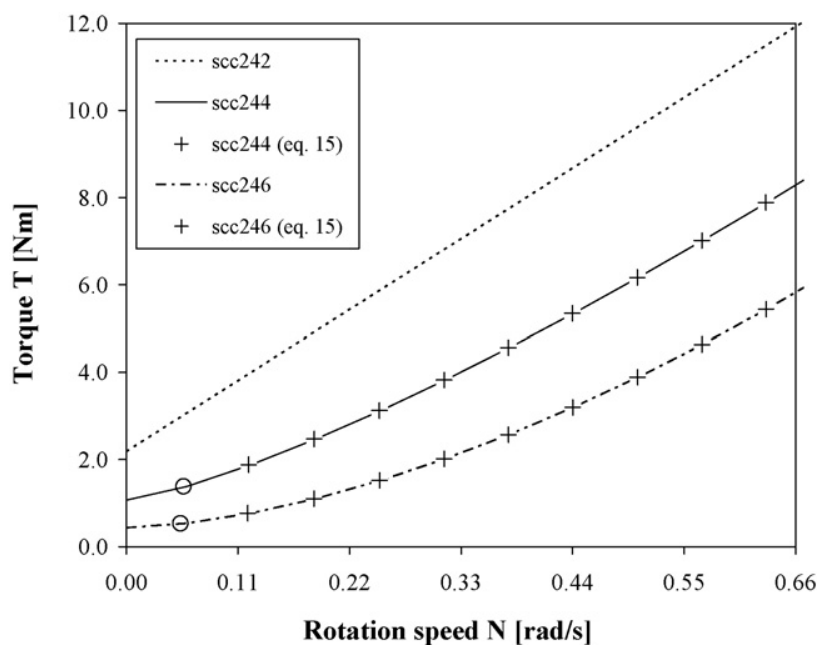

Fig. 5. Flow curves $T(N)$ for SCC24 (straight line: nonlinear least square curve fitting,+: Eq. (15), no plug, $\bigcirc$ : Eq. (15), plug).

results of other powder type SCC mixtures can be found in [8]). The resulting flow curves $T(N)$ are represented in Fig. 5.

In order to obtain the flow curves $\tau(\dot{\gamma})$ (straight lines in Fig. 6), Eqs. (19), (23) and (24) are used in order to calculate the model parameters $\tau_{0, \mathrm{HB}}, K$ and $n$. The results are given in Table 4 .

The validation of the proposed method to convert $T(N)$ into $\tau(\dot{\gamma})$ for Herschel-Bulkley fluids is done by filling in $\tau_{0, \mathrm{HB}}, K$ and $n$ in Eq. (15) and calculating the corresponding rotation speed $N$ for certain values of torque $T$. The results are represented in Fig. 5

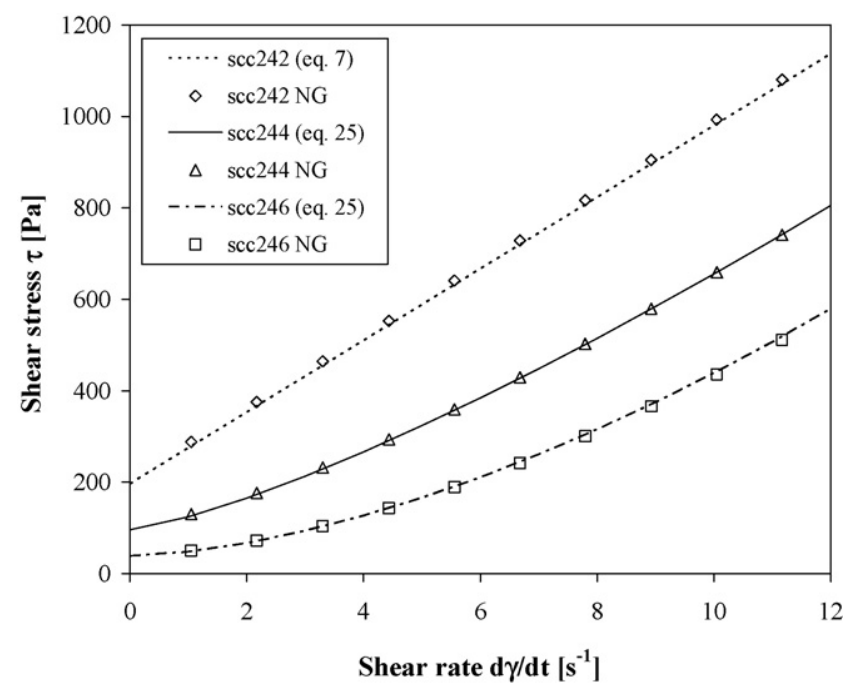

Fig. 6. Flow curves $\tau(\dot{\gamma})$ for SCC24 (straight lines) and narrow-gap (NG) approximation (point markers, according to Eqs. (26) and (27)). 
Table 4

Results for the $\tau(\dot{\gamma})$ model parameters

\begin{tabular}{lclllll}
\hline & $\tau_{0, \mathrm{HB}}(\mathrm{Pa})$ & $\Delta \tau_{0, \mathrm{HB}}(\mathrm{Pa})$ & $K\left(\mathrm{~Pa} \mathrm{~s}^{n}\right)$ & $\Delta K\left(\mathrm{Pas}^{n}\right)$ & $n(-)$ & $\Delta n(-)$ \\
\hline $\mathrm{SCC} 242$ & 196.44 & 3.47 & 78.37 & 0.58 & 1 & 0 \\
$\mathrm{SCC} 244$ & 95.72 & 5.28 & 28.24 & 0.35 & 1.30 & 0.03 \\
$\mathrm{SCC} 246$ & 38.96 & 3.21 & 8.89 & 0.15 & 1.65 & 0.03 \\
\hline
\end{tabular}

by the cross markers (+). It may be clear that Eq. (15), using the proposed $\tau(\dot{\gamma})$ model parameters, results in the same $T(N)$ curve as found by nonlinear least square curve fitting, according to Eq. (16).

For both SCC244 and SCC246, a plug was formed during the lowest rotation speed $(0.06 \mathrm{rps})$. The plug rotation speed $N_{\mathrm{p}}$, beneath which plug occurs, was found to be equal to 0.086 and $0.093 \mathrm{rps}$, respectively (see Appendix D for the determination of $N_{\mathrm{p}}$ ). In those cases, the outer boundary condition of Eq. (14) is replaced by " $r=R_{\mathrm{p}} \Rightarrow \omega \equiv \Omega_{\mathrm{o}}$ ", keeping in mind the possibility of slippage in the transition zone from viscoplastic to solid state. The results are represented in Fig. 5 by the circle markers $(\bigcirc)$. In both cases it can be concluded that no substantial slip occurred in the transition zone.

A disadvantage of both Eqs. (7) and (25) is that no information is given about the shear rate/shear stress region actually tested by the $T(N)$ measurements. For this purpose, the following approximation formulae can be used for "point by point" conversion of $T(N)$ into $\tau(\dot{\gamma})$ [35]:

$\tau=\frac{R_{\mathrm{o}}^{2}+R_{\mathrm{i}}^{2}}{4 \pi h R_{\mathrm{o}}^{2} R_{\mathrm{i}}^{2}} T$

$\dot{\gamma}=\frac{R_{\mathrm{o}}^{2}+R_{\mathrm{i}}^{2}}{R_{\mathrm{o}}^{2}-R_{\mathrm{i}}^{2}} 2 \pi N$

The results of the latter equations are shown in Fig. 6 by the point markers $(\diamond, \triangle$ and $\square)$. Note that those equations are not depending on any rheological model. However, they assume a linear velocity distribution across the gap between the two concentric cylinders, which cannot be generalized for a wide-gap concentric cylinder rheometer. When using this kind of rheometer, the assumption of a linear velocity distribution (Fig. 7) can only be used for preliminary design calculations. When more accurate calculations are required, this assumption should be kept in mind, especially when a plug is formed in the test material $[27,28]$. For a more accurate approach, the equations presented in Section 5 of this paper should be used in order to define the ultimate flow curve $\tau(\dot{\gamma})$.

It should be mentioned explicitly that the determined model parameters $\tau_{0, \mathrm{HB}}, K$ and $n$ (and thus the ultimate flow curve $\tau(\dot{\gamma}))$ are only valid for the shear rate region actually tested by the $T(N)$ measurements. Using Eq. (27) with $0.06<N<0.63 \mathrm{rps}$, a shear rate region $1.1<\dot{\gamma}<11.2 \mathrm{~s}^{-1}$ is found for the rheometer geometry used. The experiments revealed a higher probability of occurrence of experimental errors outside this shear rate region:

- Lower shear rates can introduce the appearance of plug and thus of possible slippage in the transition zone from viscoplastic to solid state (resulting in smaller torque measurements

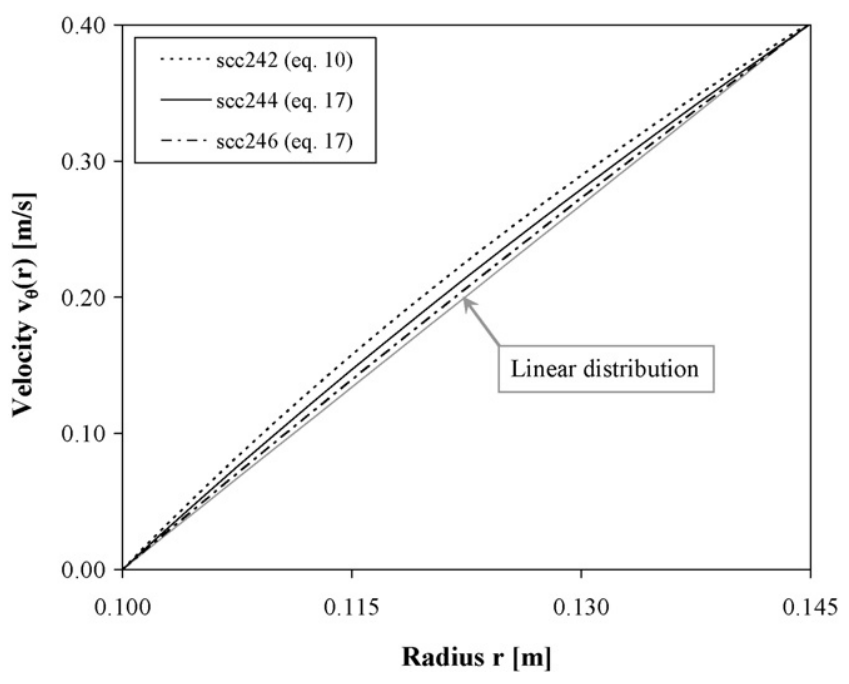

Fig. 7. Velocity distribution across the gap in the ConTec Visco5 for $N=0.44 \mathrm{rps}$.

than expected). Besides, structural reconstruction (coagulation and hydration of the cement particles) becomes more and more an influencing parameter at lower shear rates, due to the shear dependency of kinetics of dispersion and coagulation of the cement particles, possibly resulting in different rheological response and parameters obtained.

- Higher shear rates can introduce experimental errors due to e.g. particle migration (see [27] for further information on this topic).

However, the shear rate region actually tested is believed to approach the range of in situ shear behaviour of SCC during casting: $\dot{\gamma}=10 \mathrm{~s}^{-1}$ when leaving the concrete truck and $\dot{\gamma}=1 \mathrm{~s}^{-1}$ at the end of concrete flow in the mould. Note that those values are only rough estimators of actual in situ shear behaviour (assuming a $10 \mathrm{~cm}$ thick concrete layer flowing at a speed of 1 and $0.1 \mathrm{~m} / \mathrm{s}$, respectively) since to date no exact method is established in order to measure "true" in situ shear rates of concrete flow.

Higher shear rates can be needed for describing the flow behaviour of SCC during pumping, but it should be noted that a slippage layer will always be formed inside the pipe, so the shear rate is assumed to remain within certain limits. Note that shear thickening could be harmful for pumped SCC, since flowing could be impaired in the pipes. For those applications, a proper SCC mix design is required. It should be based on rheological tests, resulting in a complete flow curve in the range of the shear behaviour in the pipe in order to cover the in situ flow ability of the material.

\section{Conclusions}

An integration approach of the Couette inverse problem is proposed in order to describe the Herschel-Bulkley behaviour of self-compacting concrete, observed in a wide-gap concentric cylinder rheometer. Although the Couette inverse problem can not analytically be solved for the additive function $T=G_{\mathrm{HB}}+H_{\mathrm{HB}} N^{J}$, a reliable approach can nevertheless be found 
as the sum of the solutions of the Couette inverse problem of both subfunctions $T^{\prime}=G_{\mathrm{HB}}$ (the flow resistance) and $T^{\prime \prime}=H_{\mathrm{HB}} N^{J}$ (the power-law flow behaviour after exceeding the flow resistance). The equations needed for the conversion of the flow curve $T(N)$ into $\tau(\dot{\gamma})$ are mathematically derived in this paper. Before converting the $T(N)$ data, attention must be paid to steady state flow and to the appearance of slip or plug in order to eliminate those "experimental errors" from the test results.

Experimental validation shows that the proposed conversion method can be used to describe the rheological behaviour of powder type self-compacting concrete mixtures, commonly used in Belgium and with different superplasticizer contents added, in its fundamental rheological variables. Such approach might overcome the evaluation problems encountered in the phenomenological consistency test methods, commonly used in concrete technology.

\section{Acknowledgements}

The financial support of the Research Programme of the Research Foundation - Flanders (FWO), who made the purchase of the "ConTec Visco5" possible, is greatly acknowledged.

\section{Appendix A. Integral solution of the Couette inverse problem for a Bingham fluid}

The integral from Eq. (5) is:

$\int_{R_{\mathrm{i}}}^{R_{\mathrm{O}}}\left(\frac{T}{2 \pi r^{3} h}-\frac{\tau_{0, \mathrm{~B}}}{r}\right) \mathrm{d} r=\mu \int_{0}^{\Omega_{\mathrm{o}}} \mathrm{d} \omega(r)$

or:

$\frac{T}{2 \pi h} \int_{R_{\mathrm{i}}}^{R_{\mathrm{O}}} \frac{\mathrm{d} r}{r^{3}}-\tau_{0, \mathrm{~B}} \int_{R_{\mathrm{i}}}^{R_{\mathrm{o}}} \frac{\mathrm{d} r}{r}=\mu \Omega_{\mathrm{o}}=\mu(2 \pi N)$

and thus:

$\frac{T}{4 \pi h}\left(\frac{1}{R_{\mathrm{i}}^{2}}-\frac{1}{R_{\mathrm{o}}^{2}}\right)-\tau_{0, \mathrm{~B}} \ln \left(\frac{R_{\mathrm{o}}}{R_{\mathrm{i}}}\right)=\mu(2 \pi N)$

Rearranging the latter equation for $T$ :

$T=\frac{4 \pi h \tau_{0, \mathrm{~B}}}{\left(1 / R_{\mathrm{i}}^{2}-1 / R_{\mathrm{o}}^{2}\right)} \ln \left(\frac{R_{\mathrm{o}}}{R_{\mathrm{i}}}\right)+\frac{\mu 8 \pi^{2} h}{\left(1 / R_{\mathrm{i}}^{2}-1 / R_{\mathrm{o}}^{2}\right)} N$

Appendix B. Integral solution of the Couette inverse problem for a Herschel-Bulkley fluid

The integral from Eq. (13) is:

$\int_{R_{\mathrm{i}}}^{R_{\mathrm{o}}}\left(\left(\frac{T}{2 \pi r^{2} h K}-\frac{\tau_{0, \mathrm{HB}}}{K}\right)^{1 / n} \frac{1}{r}\right) \mathrm{d} r=\int_{0}^{\Omega_{\mathrm{o}}} \mathrm{d} \omega(r)$ or:

$\int_{R_{\mathrm{i}}}^{R_{\mathrm{o}}}\left(\left(\frac{T}{2 \pi r^{2} h K}-\frac{\tau_{0, \mathrm{HB}}}{K}\right)^{1 / n} \frac{1}{r}\right) \mathrm{d} r=\Omega_{\mathrm{o}}=2 \pi N$

First, the left-hand side of the latter equation is rewritten as:

$$
\begin{gathered}
\int_{R_{\mathrm{i}}}^{R_{\mathrm{o}}}\left(\left(\frac{\alpha T}{r^{2}}-\beta\right)^{1 / n} \frac{1}{r}\right) \mathrm{d} r \text { with } \\
\alpha=\frac{1}{2 \pi h K} \quad \text { and } \quad \beta=\frac{\tau_{0, \mathrm{HB}}}{K}
\end{gathered}
$$

To solve Eq. (B.3), the following substitution is used:

$$
\begin{aligned}
\chi & =\frac{\alpha T}{r^{2}}-\beta \Rightarrow \frac{\mathrm{d} \chi}{\mathrm{d} r}=-2 \frac{\alpha T}{r^{3}} \Rightarrow \frac{\mathrm{d} r}{r} \\
& =-\frac{r^{2} \mathrm{~d} \chi}{2 \alpha T}=-\frac{1}{2} \frac{\mathrm{d} \chi}{\chi+\beta}
\end{aligned}
$$

So, Eq. (B.3) becomes:

$-\frac{1}{2} \int_{\chi_{\mathrm{i}}}^{\chi_{\mathrm{o}}} \frac{\chi^{1 / n}}{\chi+\beta} \mathrm{d} \chi \equiv-\frac{1}{2}\left[\chi^{1 / n}\left[n-\hat{\phi}\left(-\frac{\chi}{\beta}, 1, \frac{1}{n}\right)\right]\right]_{\chi_{\mathrm{i}}}^{\chi_{\mathrm{o}}}$

where $\hat{\phi}$ denotes the "LerchPhi" function. By resubsitution of Eq. (B.5), Eq. (B.2) becomes:

$$
\begin{aligned}
& {\left[\begin{array}{l}
\left(\frac{T}{2 \pi R_{\mathrm{i}}^{2} h K}-\frac{\tau_{0, \mathrm{HB}}}{K}\right)^{1 / n}\left[n-\hat{\phi}\left(1-\frac{T}{2 \pi R_{\mathrm{i}}^{2} h \tau_{0, \mathrm{HB}}}, 1, \frac{1}{n}\right)\right]- \\
\left(\frac{T}{2 \pi R_{\mathrm{o}}^{2} h K}-\frac{\tau_{0, \mathrm{HB}}}{K}\right)^{1 / n} \quad\left[n-\hat{\phi}\left(1-\frac{T}{2 \pi R_{\mathrm{o}}^{2} h \tau_{0, \mathrm{HB}}}, 1, \frac{1}{n}\right)\right]
\end{array}\right]} \\
& =4 \pi N
\end{aligned}
$$

\section{Appendix C. Integral solution of the Couette inverse problem for an Ostwald-de Waele fluid}

The integral from Eq. (20) is:

$\int_{R_{\mathrm{i}}}^{R_{\mathrm{o}}}\left(\left(\frac{T^{\prime \prime}}{2 \pi r^{2} h K}\right)^{1 / n} \frac{1}{r}\right) \mathrm{d} r=\int_{0}^{\Omega_{\mathrm{o}}} \mathrm{d} \omega(r)$

or:

$\left(\frac{T^{\prime \prime}}{2 \pi h K}\right)^{1 / n} \int_{R_{\mathrm{i}}}^{R_{\mathrm{o}}} \frac{\mathrm{d} r}{r^{(2+n) / n}}=\Omega_{\mathrm{o}}=2 \pi N$

and thus:

$\left(\frac{T^{\prime \prime}}{2 \pi h K}\right)^{1 / n}\left(\frac{n}{2}\right)\left(\frac{1}{R_{\mathrm{i}}^{2 / n}}-\frac{1}{R_{\mathrm{O}}^{2 / n}}\right)=2 \pi N$

Rearranging the latter equation for $T^{\prime \prime}$ :

$T^{\prime \prime}=\frac{2^{2 n+1} \pi^{n+1} h K}{n^{n}\left(1 / R_{\mathrm{i}}^{2 / n}-1 / R_{\mathrm{o}}^{2 / n}\right)^{n}} N^{n}$ 


\section{Appendix D. Calculation of plug rotation speed $N_{\mathrm{p}}$ for a Herschel-Bulkley fluid}

When plug $(\dot{\gamma}=0)$ starts at the outer cylinder, the following equation holds for a Herschel-Bulkley fluid:

$R_{\mathrm{p}}^{2} \equiv R_{\mathrm{o}}^{2}=\frac{T}{2 \pi h \tau_{0, \mathrm{HB}}}$

Note that the latter equation is similar to Eq. (12), because the shear stress is only defined by the "yield" stress when $\dot{\gamma}=0$ for both a Bingham and a Herschel-Bulkley fluid.

Filling in Eq. (25) into Eq. (D.1) results in:

$$
\begin{aligned}
R_{\mathrm{o}}^{2}= & \frac{2}{\left(1 / R_{\mathrm{i}}^{2}-1 / R_{\mathrm{o}}^{2}\right)} \ln \left(\frac{R_{\mathrm{o}}}{R_{\mathrm{i}}}\right) \\
& +\frac{2^{2 n} \pi^{n} K}{\tau_{0, \mathrm{HB}}\left(1 / R_{\mathrm{i}}^{2 / n}-1 / R_{\mathrm{o}}^{2 / n}\right)^{n} n^{n}} N_{\mathrm{p}}^{\mathrm{n}}
\end{aligned}
$$

with $N_{\mathrm{p}}$ the plug rotational speed (rps), beneath which a plug is formed in the test material. From the latter equation, $N_{\mathrm{p}}$ can be calculated as:

$$
\begin{aligned}
N_{\mathrm{p}}= & \left(\frac{n}{4 \pi}\right)\left(\frac{1}{R_{\mathrm{i}}^{2 / n}}-\frac{1}{R_{\mathrm{O}}^{2 / n}}\right) \\
& \times \sqrt[n]{\left(R_{\mathrm{o}}^{2}-\frac{2}{\left(1 / R_{\mathrm{i}}^{2}-1 / R_{\mathrm{o}}^{2}\right)} \ln \left(\frac{R_{\mathrm{O}}}{R_{\mathrm{i}}}\right)\right) \frac{\tau_{0, \mathrm{HB}}}{K}}
\end{aligned}
$$

\section{References}

[1] H. Okamura, M. Ouchi, in: Å. Skarendahl, Ö. Petersson, SCC1999. Proceedings of 1st International RILEM Symposium on Self-Compacting Concrete, Stockholm, 13-14 September 1999, RILEM Publications SARL, Bagneux, pp. 3-14.

[2] BIBM, CEMBUREAU, EFCA, EFNARC and ERMCO, The European Guidelines for Self-Compacting Concrete: Specification, Production and Use, May 2005, 63 pp.

[3] G. De Schutter, Guidelines for Testing Fresh Self-Compacting Concrete, September 2005, 23 pp.

[4] C.F. Ferraris, F. de Larrard, N. Martys, in: S. Mindess, J.P. Skalny (Eds.), Materials of Science VI, Wiley-American Ceramic Society, Westerville, 2001, pp. 215-241.

[5] H.A. Barnes, The yield stress - a review or ' $\pi \alpha \nu \tau \alpha \rho \varepsilon \iota$ ' - everything flows? J. Non-Newton. Fluid Mech. 81 (1999) 133-178.

[6] C.W. Macosko, Rheology - Principles, Measurements and Applications, Wiley-VCH, New York, 1994.

[7] Ph. Coussot, Rheometry of Pastes, Suspensions and Granular Materials: Applications in Industry and Environment, John Wiley \& Sons, New Jersey, 2005.

[8] G. Heirman, et al., in: G. De Schutter, V. Boel (Eds.), SCC2007. Proceedings of 5th International RILEM Symposium on Self-Compacting Concrete, Ghent, 3-5 September 2007, RILEM Publications SARL, Bagneux, pp. 329-334

[9] D. Feys, et al., in: G. De Schutter, V. Boel (Eds.), SCC2007. Proceedings of 5th International RILEM Symposium on Self-Compacting Concrete, Ghent, 3-5 September 2007, RILEM Publications SARL, Bagneux, pp. $365-370$.
[10] C.F. Ferraris, F. de Larrard, Testing and Modelling of Fresh Concrete Rheology, NISTIR 6094, February 1998, 61 pp.

[11] F. de Larrard, C.F. Ferraris, T. Sedran, Fresh concrete: a Herschel-Bulkley material, Mater. Struct. 31 (1998) 494-498.

[12] M. Cyr, C. Legrand, M. Mouret, Study of the shear thickening effect of superplasticizers on the rheological behaviour of cement pastes containing or not mineral additives, Cem. Con. Res. 30 (2000) 1477-1483.

[13] H.A. Barnes, Shear-thickening ("dilatancy") in suspensions of nonaggregating solid particles dispersed in Newtonian liquids, J. Rheol. 33 (1989) 329-366.

[14] R.L. Hoffman, Discontinuous and dilatant viscosity behaviour in concentrated suspensions. I. Observations of a flow instability, Trans. Soc. Rheol. 16 (1972) 155-173

[15] R.L. Hoffman, Discontinuous and dilatant viscosity behaviour in concentrated suspensions. II. Theory and experimental tests, J. Colloid Interface Sci. 46 (1974) 491-506.

[16] R.L. Hoffman, Explanations for the cause of shear thickening in concentrated colloidal suspensions, J. Rheol. 42 (1998) 111-123.

[17] J.F. Brady, G. Bossis, The rheology of concentrated suspensions of spheres in simple shear flow by numerical simulation, J. Fluid Mech. 155 (1985) 105-129.

[18] J.F. Brady, G. Bossis, Stokesian dynamics, Annu. Rev. Fluid Mech. 20 (1988) 111-157.

[19] G. Bossis, J.F. Brady, The rheology of Brownian suspensions, J. Chem. Phys. 91 (1989) 1866-1874.

[20] Ó. Wallevik, The Rheology of Fresh Concrete and Its Application on Concrete With and Without Silica Fume (PhD dissertation), NTNU Trondheim, 1990, 185 pp. (only available in Norwegian).

[21] N.Q. Dzuy, D.V. Boger, Yield stress measurements for concentrated suspensions, J. Rheol. 27 (1983) 321-349.

[22] A.W. Saak, H.M. Jennings, S.P. Shah, The influence of wall slip on yield stress and viscoelastic measurements of cement paste, Cem. Con. Res. 31 (2001) 205-212.

[23] J.E. Wallevik, Thixotropic investigation on cement paste: experimental and numerical approach, J. Non-Newton. Fluid Mech. 132 (2005) 8699.

[24] M. Keentok, J.F. Milthorpe, E. O’Donovan, On the shearing zone around rotating vanes in plastic liquids: Theory and experiment, J. Non-Newton. Fluid Mech. 17 (1985) 23-25.

[25] J. Yan, A.E. James, The yield surface of viscoelastic and plastic fluids in a vane viscometer, J. Non-Newton. Fluid Mech. 70 (1997) 237-253.

[26] H.A. Barnes, Q.D. Nguyen, Rotating vane rheometry - a review, J. NonNewton. Fluid Mech. 98 (2001) 1-14.

[27] J.E. Wallevik, Rheology of Particle Suspensions, Fresh Concrete, Mortar and Cement Paste with Various Types of Lignosulfonates (PhD dissertation), NTNU Trondheim, 2003, 397 pp.

[28] G. Heirman, et al., in: J. Marchand, et al. (Eds.), Proceedings of 2nd International RILEM Symposium on Advances in Concrete through Science and Engineering, Quebec, 11-13 September 2006, RILEM Publications SARL, Bagneux, p. 327 (on CD-ROM).

[29] Y. Yeow, W. Ko, P. Tang, Solving the inverse problem of Couette viscometry by Tikhonov regularization, J. Rheol. 44 (2000) 1335-1351.

[30] C. Ancey, Solving the Couette inverse problem using a wavelet-vaguelette decomposition, J. Rheol. 49 (2005) 441-460.

[31] P. Moldenaers (Applied Rheology and Polymer Processing Section, Department of Chemical Engineering, K.U. Leuven, Belgium), personal communication, 28/02/2005.

[32] P.F.G. Banfill (School of the Built Environment, Heriot-Watt University, Scotland), personal communication, 11/04/2005.

[33] http://functions.wolfram.com/10.06.03.0020.01, 08/06/2007.

[34] H.A. Barnes, J.F. Hutton, K. Walters (Eds.), An Introduction to Rheology, Elsevier, Oxford, 1989

[35] M. Nehdi, M.A. Rahman, Effect of geometry and surface friction of test accessory on oscillatory rheological properties of cement pastes, ACI Mater. J. 101 (2004) 416-424 